Dorota Leszczyńska-Jasion, JM13714

Yaroslav Petrukhin, Vasilyi Shangin and Marcin Jukiewicz

\title{
FUNCTIONAL COMPLETENESS IN CPL via CORRESPONDENCE ANALYSIS
}

\begin{abstract}
Kooi and Tamminga's correspondence analysis is a technique for designing proof systems, mostly, natural deduction and sequent systems. In this paper it is used to generate sequent calculi with invertible rules, whose only branching rule is the rule of cut. The calculi pertain to classical propositional logic and any of its fragments that may be obtained from adding a set (sets) of rules characterizing a two-argument Boolean function(s) to the negation fragment of classical propositional logic. The properties of soundness and completeness of the calculi are demonstrated. The proof of completeness is conducted by Kalmár's method.

Most of the presented sequent-calculus rules have been obtained automatically, by a rule-generating algorithm implemented in Python. Correctness of the algorithm is demonstrated. This automated approach allowed us to analyse thousands of possible rules' schemes, hundreds of rules corresponding to Boolean functions, and to find dozens of those invertible. Interestingly, the analysis revealed that the presented proof-theoretic framework provides a syntactic characteristics of such an important semantic property as functional completeness.

Keywords: correspondence analysis, invertible rules, classical propositional logic, functional completeness, sequent calculus, automated deduction, automated rules generation.
\end{abstract}




\section{Introduction}

Correspondence analysis is Kooi and Tamminga's [13, 26] proof-theoretic approach which, originally, was developed in order to axiomatize via natural deduction systems all the truth-functional unary and binary extensions of three-valued logic $\mathbf{L P}$ (Logic of $\mathbf{P a r a d o x}$ ) [1, 24]. To be sure, this technique can be applied for other many-valued functionally incomplete logics.

In this paper, we, first, extend this method to sequent calculi. The calculi capture various fragments of CPL (Classical Propositional Logic), the whole CPL included. Second, correspondence analysis has been used here in a refined way, in order to obtain invertible rules only. The main motivation of this step is the fact that both invertibility of rules and the use of cut support proof-search. ${ }^{1}$

Last but not least, it may be observed that binary connectives can be grouped according to their syntactic characteristics provided by the invertible rules found via correspondence analysis. This characterization clearly covers with the division between primary and non-primary connectives. We have used an algorithmic approach in order to generate in an automatic manner all the possible invertible rules corresponding to particular Boolean functions. The algorithmic approach helped us to understand the observed regularities.

\section{A brief history of correspondence analysis}

- Kooi and Tamminga [13] invented correspondence analysis and used it to obtain natural deduction systems for all the unary and binary extensions of three-valued logic LP (Logic of Paradox) [1, 24].

- Tamminga [26], using correspondence analysis, presented natural deduction systems for all the unary and binary extensions of Kleene's strong three-valued logic $\mathbf{K}_{\mathbf{3}}[11,12]$.

- Petrukhin [21] formulated via correspondence analysis natural deduction systems for all the unary and binary extensions of Belnap-Dunn's four-valued logic FDE (First Degree Entailment) [2, 3, 6] supplied with Boolean negation. Petrukhin and Shangin have recently applied correspondence analysis for FDE itself [20].

\footnotetext{
${ }^{1}$ Another motivation stems from the logic of questions, but we will not elaborate on this issue here. This issue is described in another paper, "The method of Socratic proofs meets correspondence analysis" submitted to the Bulletin of the Section of Logic.
} 
- Petrukhin and Shangin [16] developed a proof-searching algorithm for natural deduction systems for all the binary extensions of LP.

- In [17], Petrukhin and Shangin extended their proof-searching technique to the case of all the binary extensions of $\mathbf{K}_{\mathbf{3}}$.

- Petrukhin [23] presented via correspondence analysis natural deduction systems for all the unary and binary extensions of Kubyshkina and Zaitsev's [14] four-valued logic LRA (Logic of Rational Agent). Besides, he generalized Kooi and Tamminga's ([13], [26]) results for a wider class of three-valued logics [22].

- Petrukhin and Shangin [18] used correspondence analysis to syntactically characterize Tomova's natural logics [27, 10].

- Petrukhin and Shangin [19] presented correspondence analysis for PWK (Paraconsistent Weak Kleene logic) [8, 4] which is Kleene's weak logic $\mathbf{K}_{\mathbf{3}}^{\mathbf{w}}[11,12]$ with two designated values.

Let us mention Segerberg's paper [25] where one may find a predecessor of the method of correspondence analysis. In fact, Segerberg has already used the very idea of correspondence between truth table entries and inference rules, but he has not emphasized it via a special definition in contrast to Kooi and Tamminga (see Definition 1 of single entry correspondence). He developed a general way of producing sound and complete natural deduction rules for all Boolean $n$-ary functions. However, his rules generally are not invertible. Hence, they are not suitable for our purposes.

\section{Correspondence analysis for CPL}

Notation. Let $\mathscr{P}$ be a countably infinite set $\left\{p_{1}, p_{2}, p_{3}, \ldots\right\}$ of propositional variables and let $\mathscr{B}=\left\{\circ_{\perp}, \wedge, \not \rightarrow, \circ_{1}, \nvdash, \circ_{2}, \underline{\vee}, \vee, \downarrow, \equiv, \circ_{\neg 2}, \leftarrow, \circ_{\neg 1}\right.$, $\left.\rightarrow, \uparrow, \circ_{\top}\right\}$ be a set of binary operators, where:

\begin{tabular}{|c|c||c|c|c|c|c|c|c|c|}
\hline$A$ & $B$ & $\circ_{\perp}$ & $\wedge$ & $\not \rightarrow$ & $\circ_{1}$ & $\nvdash$ & $\circ_{2}$ & $\underline{\vee}$ & $\vee$ \\
\hline \hline 1 & 1 & 0 & 1 & 0 & 1 & 0 & 1 & 0 & 1 \\
\hline 1 & 0 & 0 & 0 & 1 & 1 & 0 & 0 & 1 & 1 \\
\hline 0 & 1 & 0 & 0 & 0 & 0 & 1 & 1 & 1 & 1 \\
\hline 0 & 0 & 0 & 0 & 0 & 0 & 0 & 0 & 0 & 0 \\
\hline
\end{tabular}




\begin{tabular}{|c|c||c|c|c|c|c|c|c|c|}
\hline$A$ & $B$ & $\downarrow$ & $\equiv$ & $\circ_{\neg 2}$ & $\leftarrow$ & ${ }_{\neg 1}$ & $\rightarrow$ & $\uparrow$ & ${ }^{\top}$ \\
\hline \hline 1 & 1 & 0 & 1 & 0 & 1 & 0 & 1 & 0 & 1 \\
\hline 1 & 0 & 0 & 0 & 1 & 1 & 0 & 0 & 1 & 1 \\
\hline 0 & 1 & 0 & 0 & 0 & 0 & 1 & 1 & 1 & 1 \\
\hline 0 & 0 & 1 & 1 & 1 & 1 & 1 & 1 & 1 & 1 \\
\hline
\end{tabular}

Let $\mathscr{L}_{\neg}^{\circ}$ be propositional language with the alphabet $\langle\mathscr{P}, \mathscr{B}, \neg,()$,$\rangle . We$ define the set $\mathscr{F}_{\neg}^{\circ}$ of all $\mathscr{L}_{\neg}^{\circ}$ 's formulas in a standard way.

Let us recall some well-known facts concerning functional completeness (see [29]). A binary connective $\circ$ such that $\{\neg, \circ\}$ is functionally complete, will be called primary (see [7, p. 13]), other binary connectives are called non-primary. Primary connectives of $\mathscr{L}_{\neg}^{\circ}$ are: $\wedge, \nrightarrow, \leftarrow, \vee, \downarrow, \leftarrow, \rightarrow, \uparrow$. Among the non-primary connectives we will distinguish trivial (for lack of a better name) and non-trivial ones. Connective $\circ$ is trivial if the only 2argument Boolean functions that can be defined by $\{\neg, \circ\}$ are expressed by $A \circ B, B \circ A, \neg(A \circ B)$ and $\neg(B \circ A)$. Among the non-primary connectives, $\circ_{\top}, \circ_{1}, \circ_{2}, \circ_{\neg 2}, \circ_{\neg 1}, \circ_{\perp}$ are trivial: $\circ_{\top}$ together with $\neg$ defines $\circ_{\perp}$, similarly $\circ_{\perp}$ defines $\circ_{\top}$, any of $\circ_{1}, \circ_{2}, \circ_{\neg 2}, \circ_{\neg 1}$ together with $\neg$ defines all of $\circ_{1}, \circ_{2}$, $\circ_{\neg 2}, \bigcirc_{\neg 1}$. The remaining connectives (non-primary and non-trivial) are $\underline{\vee}$ and $\equiv$. Each of them, together with $\neg$, produces $\left\{\neg, \underline{\vee}, \equiv, \circ_{\perp}, \circ_{\top}\right\}$.

Why do we consider such a rich language? Mainly because correspondence analysis invites and supports this step.

Let $\circ$ stand for any of the 16 binary connectives listed above. Let $f_{\neg}$ and $f_{\circ}$ be, respectively, truth tables of truth functional operators $\neg$ and $\circ$. Let $x, y, z \in\{0,1\}$ and $f_{\circ}(x, y)=z$ be an $f_{\circ}$ 's entry such that if $v(A)=x$ and $v(B)=y$, then $v(A \circ B)=z$, where $v$ is an arbitrary valuation and $A, B \in \mathscr{F}_{\neg}^{\circ}$.

The idea of correspondence. Let $A \in \mathscr{F}_{\neg}^{\circ}$ and $X \subseteq \mathscr{F}_{\neg}^{\circ}$. If it is true that a natural-deduction rule of the form $X / A$ is semantically correct iff $f_{\circ}(x, y)=z$ is true of $\circ$, then we say that there is a correspondence between the rule and the entry.

Sequents are introduced as expressions of language $\mathscr{L}_{\neg \Rightarrow}^{\circ}$ which is built upon $\mathscr{L}_{\neg}^{\circ}$. Formally, $\mathscr{L}_{\neg \Rightarrow}^{\circ}$ is a language with the alphabet of language $\mathscr{L}_{\neg}^{\circ}$ enriched with ' $\Rightarrow$ ' (the sequent arrow) and the comma ','. The only category of a well-formed expression of $\mathscr{L}_{\neg \Rightarrow}^{\circ}$ is that of a sequent of $\mathscr{L}_{\neg \Rightarrow}^{\circ}$, which is an expression of the form:

$$
\Gamma \Rightarrow \Delta
$$


where $\Gamma$ and $\Delta$ are finite, possibly empty multisets of formulas of $\mathscr{L}_{\neg}^{\circ}$. If $\Gamma=A_{1}, \ldots, A_{n}, \Delta=B_{1}, \ldots, B_{k}$, then a comma occurring in the antecedent and the succedent of (2.1) may be thought of as the sign of sum of multisets. Expression ' $A, \Gamma \Rightarrow \Delta^{\text {' }}$ is to be understood as:

$$
A, A_{1}, \ldots, A_{n} \Rightarrow B_{1}, \ldots, B_{k}
$$

and similarly in analogous contexts.

Sequent calculus for the negation fragment of CPL. Our aim is to find invertible rules corresponding to particular binary connectives. These will be added to a "sequent base", that is the rules for the negation fragment of classical logic together with the structural rule of the cut, which is the only branching rule of the system.

$$
\begin{gathered}
(\text { Ax }) \quad A, \Gamma \Rightarrow \Delta, A \\
\frac{A, \Gamma \Rightarrow \Delta}{\Gamma \Rightarrow \Delta, \neg A}(\Rightarrow \neg) \quad \frac{\Gamma \Rightarrow \Delta, A}{\neg A, \Gamma \Rightarrow \Delta}(\neg \Rightarrow) \\
\frac{\Gamma \Rightarrow \Delta, A \quad A, \Gamma \Rightarrow \Delta}{\Gamma \Rightarrow \Delta} \text { (cut) }
\end{gathered}
$$

The symbol ' $\models$ ' stands for entailment in both languages: $\mathscr{L}_{\neg}^{\circ}$ and $\mathscr{L}_{\neg}^{\circ}$, but, as we shall see, this should not lead to confusion. If $\Gamma=A_{1}, \ldots, A_{n}$ is a finite multiset of formulas of $\mathscr{L}_{\neg}^{\circ}$, then ' $\wedge \Gamma^{\prime}$ stands for a generalized conjunction of $\Gamma$, that is, a formula of the form:

$$
A_{1} \wedge\left(A_{2} \wedge \ldots \wedge A_{n}\right)
$$

and similarly for ' $\bigvee \Gamma$ ' (a generalized disjunction). ${ }^{2}$ If $v$ is a valuation, then we say that sequent $(2.1)$ is true under $v$ iff $v(\bigwedge \Gamma)=1$ yields $v(\bigvee \Delta)=1$. Hence it follows that if (2.1) is true under every valuation, then $\bigwedge \Gamma \models \bigvee \Delta$. Finally, the inscription:

$$
\Gamma \Rightarrow \Delta \models \Theta \Rightarrow \Lambda
$$

means that for every valuation $v$, if sequent $\Gamma \Rightarrow \Delta$ is true under $v$, then sequent $\Theta \Rightarrow \Lambda$ is true under $v$. Let us observe that in the case of both languages: $\mathscr{L}_{\neg \Rightarrow}^{\circ}$ and $\mathscr{L}_{\neg}^{\circ}, \models$ amounts to transmission of truth.

\footnotetext{
${ }^{2}$ Since $\Gamma$ is a multiset, not a sequence, this is not an unequivocal description. However, since $\wedge, \vee$ are commutative and associative in classical logic, we may assume that the formulas $A_{1}, \ldots, A_{n}$ are given in any order-e.g. alphabetical one.
} 
For our case we need the following version of Kooi and Tamminga's definition of a single entry correspondence presented in [13, Definition 2.1] and [26, Definition 1].

Definition 1 (Single entry correspondence). Let $\Gamma, \Delta, \Theta, \Lambda$ stand for finite, possibly empty multisets of formulas of $\mathscr{L}_{\neg}^{\circ}$. Let $x, y, z \in\{0,1\}$. Then we say that the truth table entry $f_{\circ}(x, y)=z$ is characterized by an inference scheme $\Gamma \Rightarrow \Delta / \Theta \Rightarrow \Lambda$, if

$$
f_{\circ}(x, y)=z \text { if and only if } \Gamma \Rightarrow \Delta \models \Theta \Rightarrow \Lambda .^{3}
$$

As we have explained in the introduction, our aim was to find not only schemes that correspond to particular entries, but ones that, when inverted, still correspond to an entry (not necessarily the same one). In other words, we have found inference schemes such that if $f_{\circ}(x, y)=z$, where $x, y, z \in$ $\{0,1\}$, is characterized by $\Gamma \Rightarrow \Delta / \Theta \Rightarrow \Lambda$, then there are $x^{\prime}, y^{\prime}, z^{\prime} \in\{0,1\}$ such that $f_{\circ}\left(x^{\prime}, y^{\prime}\right)=z^{\prime}$ is characterized by $\Theta \Rightarrow \Lambda / \Gamma \Rightarrow \Delta$. What is more, in this account the rules are not only invertible, but they will be actually inverted and used in both directions. ${ }^{4}$ The reason for this is that, as we shall see, both directions are actually needed.

By invertibility of a rule we mean the fact that the correctness of the conclusion yields the correctness of the premise. More specifically, we say that rule $\Gamma \Rightarrow \Delta / \Theta \Rightarrow \Lambda$ is:

- sound iff for each valuation $v$, if $\Gamma \Rightarrow \Delta$ is true under $v$, then $\Theta \Rightarrow \Lambda$ is true under $v$;

- invertible iff for each valuation $v$, if $\Theta \Rightarrow \Lambda$ is true under $v$, then $\Gamma \Rightarrow \Delta$ is true under $v$.

In the case of branching rules, like (cut), soundness amounts to the fact that for each $v$, if both premises are true under $v$, then the conclusion is true under $v$. In turn, invertibility of such a rule amounts to the fact that for each $v$, if the conclusion is true under $v$, then so are both premises. The context-sharing version of (cut) that we have adopted is sound and invertible. So are the rules for negation.

\footnotetext{
${ }^{3}$ Note that in the original formulation, Kooi and Tamminga deal with a three-valued logic and natural deduction rules. Thus, in their definition, $f_{\circ}(x, y)=z$ is characterized by an inference scheme $\Gamma / A$, if $f_{\circ}(x, y)=z$ if and only if $\Gamma \models A$. However, Kooi and Tamminga considered the unary connectives as well.

${ }^{4}$ As in the paradigm introduced by Došen [5]. However, our rules differ from that of Došen. Needless to say, so do our research goals.
} 
Let us introduce some additional terminology: in structural proof theory it is common to use the notions of an active formula (active formulas) and a primary formula of a rule. A formula is called active in a rule, if it is distinguished in its premise, and it is called primary, if it is distinguished in the conclusion. The remaining formulas form the context of the premise or conclusion, respectively. Since we are going to invert the rules, the distinction between active and primary may be misleading, hence we will use the term: distinguished formula of a rule. Hence a formula distinguished in a scheme is one that is either active or primary.

The following rules have been found by a human researcher. The rules have no contexts, that is, only distinguished formulas are displayed. Note that the numbers in parentheses $((01),(02)$ etc.) mark pairs of invertible rules.

Theorem 1. Let $A, B \in \mathscr{F}_{\neg}^{\circ}$. Then:

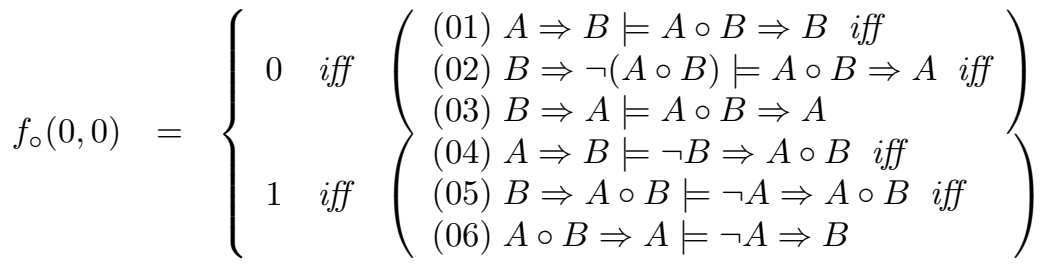

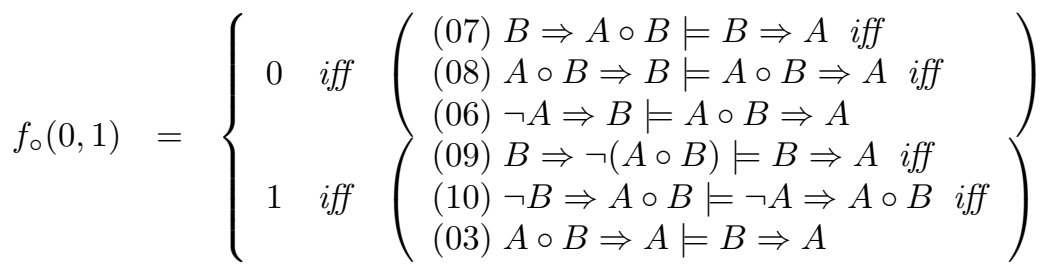

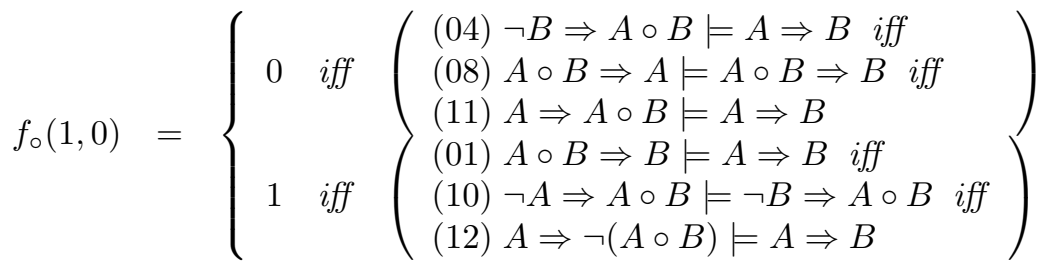




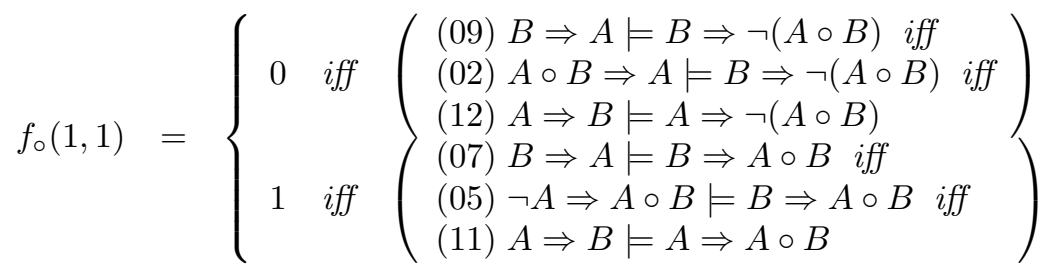

Proof: As an example, we prove the case $f_{\circ}(0,0)=1$ iff (04) $A \Rightarrow B=$ $\neg B \Rightarrow A \circ B$. (All the remaining cases will follow from a result proved below, see Lemma 1, page 57.)

Suppose $A \Rightarrow B \forall \forall \neg B A \circ B$. Then there is a valuation $v$ such that if $v(A)=1$, then $v(B)=1 ; v(\neg B)=1$ and $v(A \circ B)=0$. Then $v(B)=0$. Therefore, $v(A)=0$. Thus, $f_{\circ}(0,0) \neq 1$. So, if $f_{\circ}(0,0)=1$, then $A \Rightarrow B=\neg B \Rightarrow A \circ B$.

Suppose $A \Rightarrow B \models \neg B \Rightarrow A \circ B$. Then, for each valuation $v$, if (if $v(A)=1$, then $v(B)=1$ ), then (if $v(B)=0$, then $v(A \circ B)=1$ ). Let " $v(A)=1$ " be $X, " v(B)=1$ " be $Y$, and " $v(A \circ B)=1$ " be $Z$. Then we obtain $(X \rightarrow Y) \rightarrow(\neg Y \rightarrow Z)$. By classical logic, we obtain if $(X \rightarrow Y) \rightarrow(\neg Y \rightarrow Z)$ is true, then $(\neg X \wedge \neg Y) \rightarrow Z$ is true. Thus, for each valuation $v$, if $v(A)=v(B)=0$, then $v(A \circ B)=1$, i.e. $f_{\circ}(0,0)=1$.

Theorem 1 provides us with schemes of rules which may be used to build sequent calculi. Each scheme has exactly four distinguished formulas, always one formula in the antecedent of a sequent and one in the succedent. Unfortunately, the above rules are not sufficient for the whole CPL. Each fragment of CPL that may be expressed without the use of the primary connectives may be captured by the above rules, but they fail to characterize the primary connectives.

We give an example. Suppose o to be equivalence (三). Using Theorem 1 , we can characterize it by three different pairs of invertible rules. Consider $f_{\equiv}(0,0)=1$. We can choose an inference scheme for $f_{\equiv}(0,0)=1$ from the following pairs of inference schemes: (04), (05), and (06). Suppose we choose (04). Then we have an inference scheme for $f_{\equiv}(1,0)=0$ as well. The remaining entries characterizing $\equiv$ are $f_{\equiv}(1,1)=1$ and $f_{\equiv}(0,1)=0$, and the only rule that corresponds to both of them is $(07)$. Thus for $\equiv$ we may adopt the following rules: 


$$
R_{\equiv}^{(04)} \frac{A, \Gamma \Rightarrow \Delta, B}{\neg B, \Gamma \Rightarrow \Delta, A \equiv B} \quad R_{\equiv}^{(07)} \frac{B, \Gamma \Rightarrow \Delta, A}{\overline{B, \Gamma \Rightarrow \Delta, A \equiv B}}
$$

Double lines indicate that the rules work both ways. Surprisingly, the two rules (or two pairs of rules, to be more specific) are sufficient to build a sequent calculus for the fragment of $\mathbf{C P L}$ expressed with $\neg$, 三; one can see this from the details of the completeness proof presented in Section 4 .

We could have obtained adequate characteristics of $\equiv$ also by the pair (05) and (08), or by the pair (06) and (11). The situation is quite similar when $\underline{\vee}$ is concerned. In the case of the other non-primary connectives, there is always exactly one "match" of 2 rules that together correspond to the 4 entries characterizing the connective. Table 1 presents these "matches".

Table 1. Rules for non-primary connectives

\begin{tabular}{c|c|c|c}
\hline$A \circ_{\perp} B$ & $A \circ_{1} B$ & $A \circ_{2} B$ & $A \vee B$ \\
\hline$(02),(08)$ & $(01),(07)$ & $(03),(11)$ & $(01),(09)$ \\
& & & $\begin{array}{l}(02),(10) \\
(03),(12)\end{array}$ \\
\hline$A \equiv B$ & $A \circ_{\neg 2} B$ & $A \circ_{\neg 1} B$ & $A \circ \top B$ \\
\hline$(04),(07)$ & $(06),(12)$ & $(04),(09)$ & $(05),(10)$ \\
$(05),(08)$ & & & \\
$(06),(11)$ & & &
\end{tabular}

What about the primary connectives? Unfortunately, there never is a match among (01)-(12). Consider $\vee$ as an example. In order to gain full characteristics of the connective via our invertible rules, we need a rule corresponding to $f_{\circ}(1,1)=1$, that is, one of $(07),(05)$, (11); but (07) corresponds also to $f_{\circ}(0,1)=0,(05)$ corresponds also to $f_{\circ}(0,0)=1$, and (11) corresponds also to $f_{\circ}(1,0)=0$, so none of them fits $\vee$. To be sure, using Theorem 1 one can obtain non-invertible rules for the non-primary connectives, but the above invertible rules are not sufficient. However, we have found what follows:

Theorem 2. Let $A, B \in \mathscr{F}_{\neg}^{\circ}$. Then: 


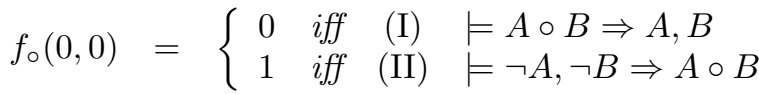

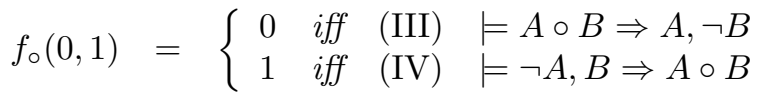

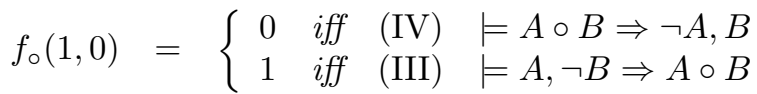

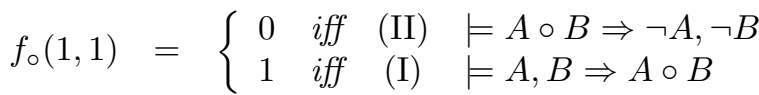

Proof: As an example, we consider the case $f_{\circ}(0,0)=0$. Suppose $\not$ $A \circ B \Rightarrow A, B$. Then there is a valuation $v$ such that $v(A \circ B)=1$ while $v(A)=v(B)=0$. Thus, $f_{\circ}(0,0) \neq 0$.

Suppose $=A \circ B \Rightarrow A, B$. Then, for each valuation $v$, if $v(A \circ B)=1$, then $v(A)=1$ or $v(B)=1$. Thus, if $v(A)=v(B)=0$, then $v(A \circ B)=0$, i.e. $f_{\circ}(0,0)=0$.

There are two ways to obtain the sequent rules from the inferences schemes presented in Theorem 2. The first one supposes that we treat these inference schemes as axioms. We use Roman numerals in parentheses ((I), (II) etc.) in order to mark pairs of axioms. In fact, axioms from the same pair are invertible in the following sense:

Definition 2. Let $\Gamma \Rightarrow \Delta$ be a sequent of language $\mathscr{L}_{\neg}^{\circ}$. We call $\Gamma \Rightarrow \Delta$ invertible, if it is both the case that $\models \Gamma \Rightarrow \Delta$ and $\models \Delta \Rightarrow \Gamma$.

Our invertible axioms have the following form:

$$
\begin{array}{cl}
A_{\circ \uparrow}^{(\mathrm{I})} A, B, \Gamma \Rightarrow \Delta, A \circ B & A_{\circ \downarrow}^{(\mathrm{I})} A \circ B, \Delta \Rightarrow \Gamma, A, B \\
A_{\circ \uparrow}^{(\mathrm{II})} \neg A, \neg B, \Gamma \Rightarrow \Delta, A \circ B & A_{\circ \downarrow}^{(\mathrm{II})} A \circ B, \Delta \Rightarrow \Gamma, \neg A, \neg B \\
A_{\circ \uparrow}^{(\mathrm{III})} A, \neg B, \Gamma \Rightarrow \Delta, A \circ B & A_{\circ \downarrow}^{(\mathrm{III})} A \circ B, \Delta \Rightarrow \Gamma, A, \neg B \\
A_{\circ \uparrow}^{(\mathrm{IV})} \neg A, B, \Gamma \Rightarrow \Delta, A \circ B & A_{\circ \downarrow}^{(\mathrm{IV})} A \circ B, \Delta \Rightarrow \Gamma, \neg A, B
\end{array}
$$

Combining these axioms with the invertible rules which were obtained by Theorem 1, as well as the axioms and the rules for the negation fragment of CPL, we can obtain sequent calculi for all the extensions of the negation fragment of CPL by binary connectives - both primary and non-primary. See the table below. 


\begin{tabular}{|r|l|}
\hline (I) and (08) & $A \circ B=A \wedge B$ \\
\hline (I) and (10) & $A \circ B=A \vee B$ \\
\hline (II) and (08) & $A \circ B=A \downarrow B$ \\
\hline (II) and (10) & $A \circ B=A \uparrow B$ \\
\hline (III) and (02) & $A \circ B=A \not \neg B$ \\
\hline (III) and (05) & $A \circ B=A \leftarrow B$ \\
\hline (IV) and (02) & $A \circ B=A \nvdash B$ \\
\hline (IV) and (05) & $A \circ B=A \rightarrow B$ \\
\hline
\end{tabular}

The second way is to transform these inference schemes into invertible rules which have the following form:

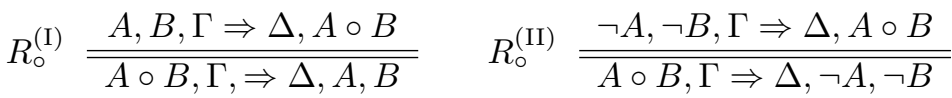

$$
\begin{aligned}
& R_{\circ}^{(\mathrm{III})} \frac{A, \neg B, \Gamma \Rightarrow \Delta, A \circ B}{A \circ B, \Gamma \Rightarrow \Delta, A, \neg B} \quad R_{\circ}^{(\mathrm{IV})} \frac{\neg A, B, \Gamma \Rightarrow \Delta, A \circ B}{A \circ B, \Gamma \Rightarrow \Delta, \neg A, B}
\end{aligned}
$$

We combine these invertible rules with the ones which were obtained by Theorem 1 in the same way we did above for the case of invertible axioms. This approach is sound due to the following consequence of Theorem 2 . Note that there is a general method due to Indrzejczak [9] of the transformation of the axioms into the sequent rules which, surely, can be applied in our case as well.

Corollary 1. Let $A, B \in \mathscr{F}_{\neg}^{\circ}$. Then:

$$
\begin{aligned}
& f_{\circ}(0,0)=\left\{\begin{array}{llll}
0 & \text { iff } & \text { (I) } & A, B \Rightarrow A \circ B=A \circ B \Rightarrow A, B \\
1 & \text { iff } & \text { (II) } & A \circ B \Rightarrow \neg A, \neg B=\neg A, \neg B \Rightarrow A \circ B
\end{array}\right. \\
& f_{\circ}(0,1)=\left\{\begin{array}{llll}
0 & \text { iff } & \text { (III) } & A, \neg B \Rightarrow A \circ B=A \circ B \Rightarrow A, \neg B \\
1 & \text { iff } & \text { (IV) } & A \circ B \Rightarrow \neg A, B=\neg A, B \Rightarrow A \circ B
\end{array}\right. \\
& f_{\circ}(1,0)=\left\{\begin{array}{llll}
0 & \text { iff } & \text { (IV) } & \neg A, B \Rightarrow A \circ B=A \circ B \Rightarrow \neg A, B \\
1 & \text { iff } & \text { (III) } & A \circ B \Rightarrow A, \neg B=A, \neg B \Rightarrow A \circ B
\end{array}\right. \\
& f_{\circ}(1,1)=\left\{\begin{array}{llll}
0 & \text { iff } & \text { (II) } & \neg A, \neg B \Rightarrow A \circ B=A \circ B \Rightarrow \neg A, \neg B \\
1 & \text { iff } & \text { (I) } & A \circ B \Rightarrow A, B=A, B \Rightarrow A \circ B
\end{array}\right.
\end{aligned}
$$

There is a clear difference between the rules considered in Theorem 1 (only non-primary connectives) and Corollary 1 (primary connectives). In the former case, each rule is composed of two sequents with exactly two distinguished formulas each, whereas in the latter case the sequents have exactly three distinguished formulas each. The question: why it is the case, 
seemed puzzling. Is it a feature of the primary / non-primary connectives, or maybe we were not ingenious enough to find some counter-examples to this apparent regularity? In order to answer this question, a human researcher decided to check all the possible schemes automatically.

\section{More rules: algorithmic approach}

In this section we present Algorithm 1, designed to find all cases of sequentcalculus rules corresponding to the particular entries. The algorithm was implemented in Python.

Language for the purpose of the implementation. Vocabulary consists of the following signs: $A, B, \neg, \circ,($,$) . Formulas are expressions of$ the form: $A, B, \neg A, \neg B, A \circ B, \neg(A \circ B)$. Inference schemes, or simply schemes, are pairs $\left(L_{1}, L_{2}\right)$ such that $L_{1}$ is a list of formulas and $L_{2}$ is a list of formulas. Lists are treated as sets, that is, repetitions and order are neglected. (However, let us recall that in a pair the order matters.) There are exactly 63 different non-empty lists containing some (possibly all) formulas. Hence there are exactly $3969(=63 \cdot 63)$ different schemes combined from the lists.

If a list is of the form $(L, L)$, that is, the conclusion is identical to the premise, then the scheme is called $i d$-trivial. There are exactly 3906 (3969$63)$ different schemes which are not id-trivial. The rules are written in the file RulesAll.txt. All the files mentioned in this Section are available on https://github.com/mjukiewicz/Correspondence-Analysis in the txt format together with a tex source.

Interpretation of lists and sequents. The intended reading of a single list is a disjunction. Sequents may be used to eliminate the use of negation. Hence, e.g., a list containing ' $A, \neg B, A \circ B$ ' represents: the disjunction ' $A \vee(\neg B) \vee(A \circ B)$ '; and also the sequent ' $B \Rightarrow A, A \circ B$ '. We deal with the nice and simple $\mathbf{C P L}$, and the deduction theorem assures the correspondence between the use of negation and disjunction (i.e. implication) and the sequent arrow. It is convenient to analyse lists without the sequent arrow, but to display the schemes as sequent rules.

Trivial cases of rules. If the first list of a scheme is contained in the second list, i.e., each formula from the premise is present in the conclusion, then the scheme is correct for trivial reasons, which excludes correspondence with an entry. (For exactly the same reason validity of a formula of the form ' $A \circ B \rightarrow((A \circ B) \vee C)$ ' does not depend on the properties of ०.) 
Another trivial case is that of a non-falsifiable conclusion. Again, if correctness of a scheme follows from the fact that the conclusion is an axiom, that is, of the form ' $\Gamma, C \Rightarrow C, \Delta$ ', then the correctness is not connected to an entry.

Algorithm 1 generates all the schemes corresponding to the following entry: ' $f(0,0)=0$ '.

The lists for the remaining 7 entries are obtained by algorithms constructed as Algorithm 1 but with a suitable modification of lines 11-13, as indicated in Table 2 on page 59. For each entry a separate file has been generated, their names are indicated in Table 2. Each file contains exactly 68 schemes of rules.

\section{Correctness of the algorithm}

The following Lemma 1 establishes that the algorithm is correct, that is, that the list of schemes which were "not rejected" is exactly the list of schemes that correspond to the relevant entry. Lemma 1 will also yield soundness of our sequent calculi.

Lemma 1. Let $\mathcal{L}_{i j k}$ stand for the list of schemes generated by Algorithm $1+$ Table 2 , suited to the entry $f_{\circ}(i, j)=k$. Let $S \in \mathcal{L}_{i j k}$. Entry $f_{\circ}(i, j)=k$ holds iff $S$ is semantically correct.

Proof: The proof goes by cases. Let $i=j=k=0$ and $S=\left(L_{1}, L_{2}\right)$.

$(\rightarrow)$ Suppose that entry $f_{\circ}(0,0)=0$ holds for ' $\circ$ ', but the scheme is not correct, that is, it is possible to assign value 1 to at least one element of $L_{1}$ and value 0 to every element of $L_{2}$. Let $H$ stand for the element of $L_{1}$ which is true under this purported countervaluation $v$. In the while-loop (lines 9-20) we arrive at an iteration with $H$ as the head of the list. Then $H \notin L_{2}$, for otherwise the conclusion of $S$ would be true under $v$. Scheme $S$ has not been rejected, so the if-then-else conjunctive condition described in lines 11,12,13 of Algorithm 1 has failed. It means that at least one of the three conjuncts failed:

If the one described in line 11 failed, then: $H=\neg A$, and $B, \neg(A \circ B)$ are elements of $L_{2}$. Then $v(A)=0, v(B)=0$, whereas $v(A \circ B)=1$, which contradicts entry $f_{\circ}(0,0)=0$.

If 12 failed, then: $H=\neg B$, so $v(B)=0, A$ and $\neg(A \circ B)$ are elements of $L_{2}$, and thus are both assigned 0 , which contradicts the entry. Similarly if 13 failed. 


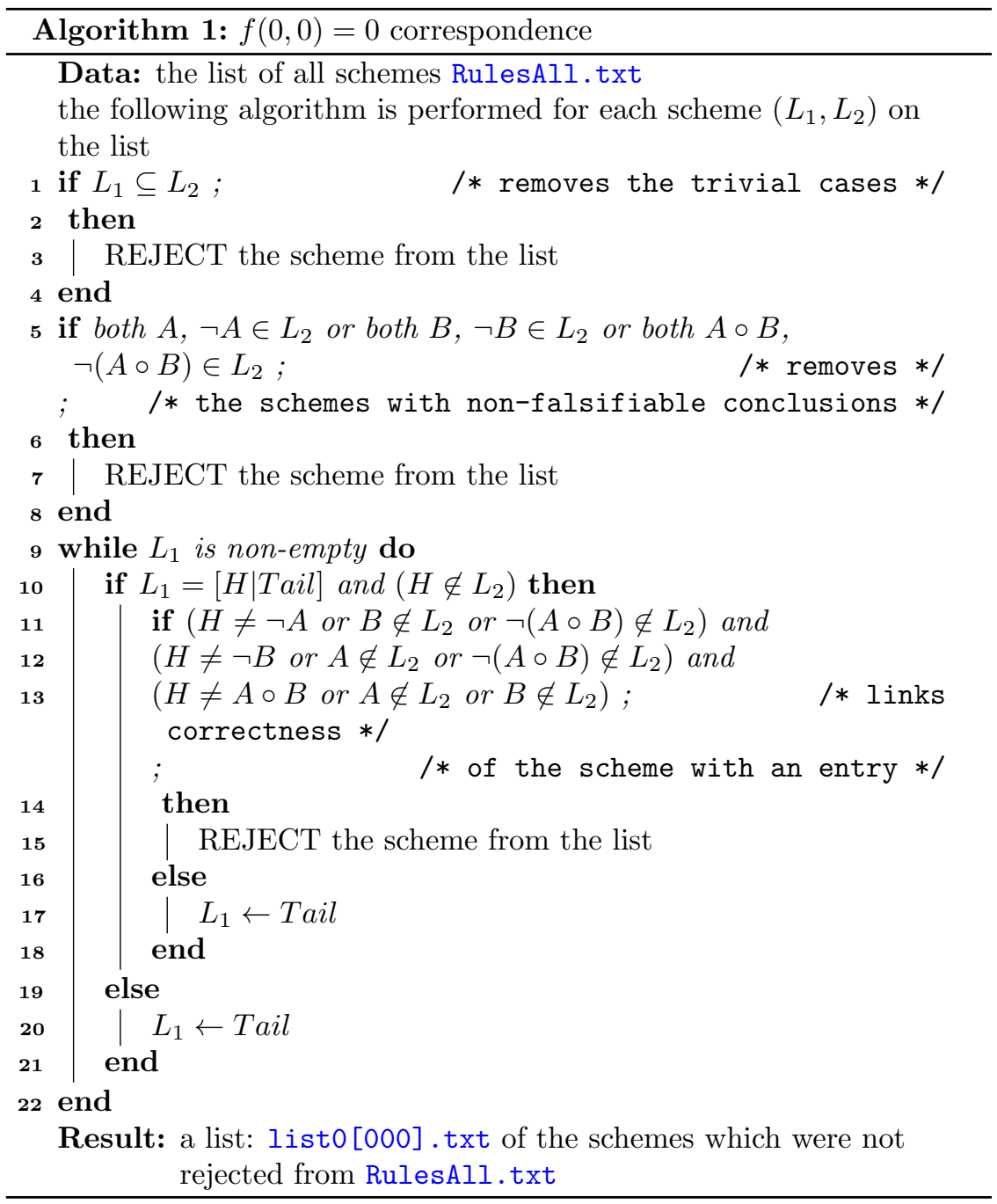

$(\leftarrow)$ Suppose that the scheme is correct, although $f_{\circ}(0,0) \neq 0$. Since the scheme is not rejected in lines 1-4 of the algorithm, there is a formula $H$ in $L_{1}$ which is not an element of $L_{2}$. We consider what happens when in the 
Table 2. modifications of Algorithm 1 for the other entries

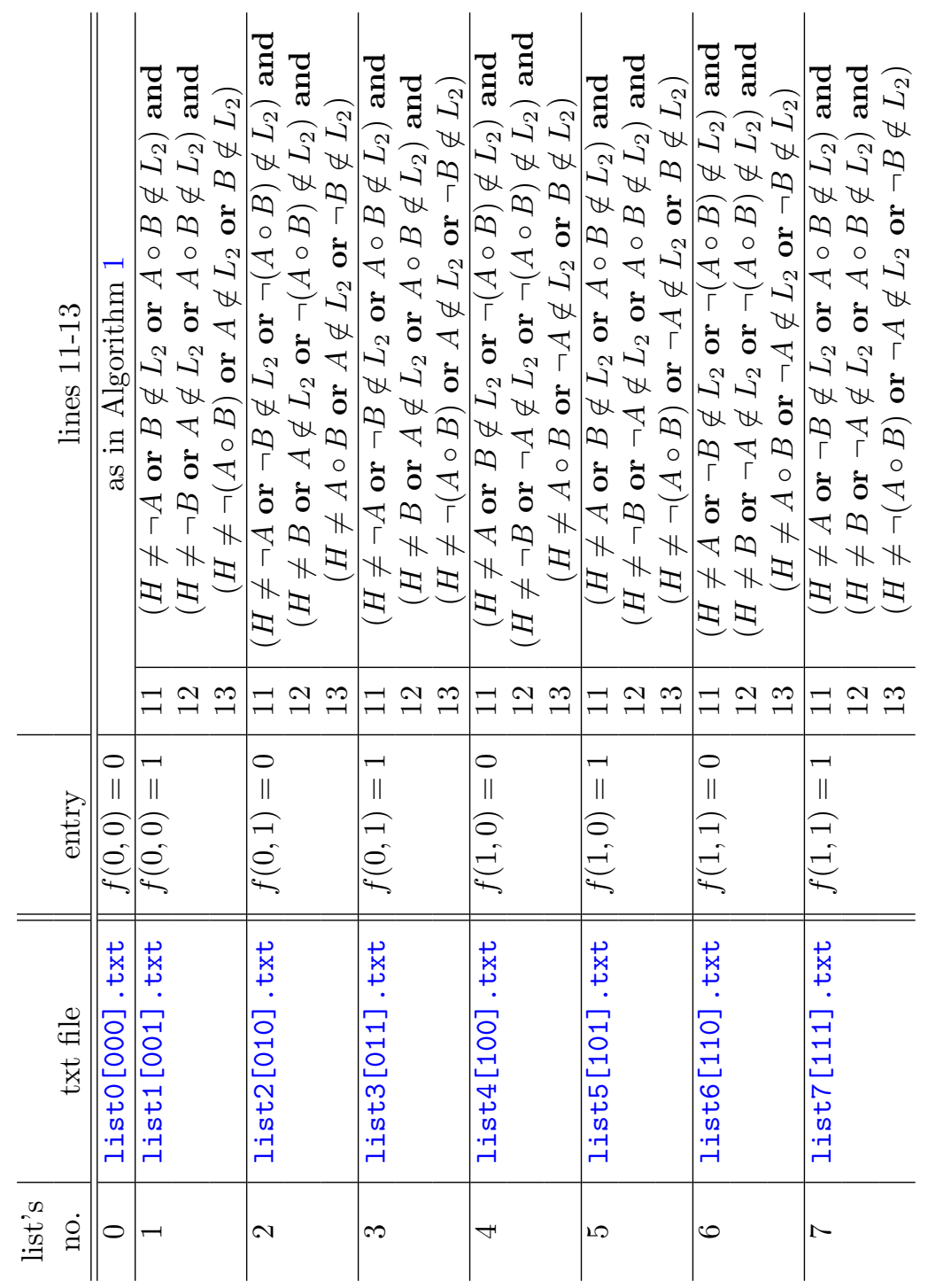


while-loop we arrive at $H$ in the head of $L_{1}$. The scheme is not rejected, so at least one of 11-13 fails. If 11 fails, then $H$ is of the form $\neg A$, and both $B$ and $\neg(A \circ B)$ are elements of $L_{2}$. Since the scheme has not been rejected in 5 -8, list $L_{2}$ contains neither $\neg B$ nor $A \circ B$. We already know that $\neg A \notin L_{2}$, therefore, except for $B$ and $\neg(A \circ B), L_{2}$ can contain only $A$, that is, $L_{2}$ is either composed of $A, B, \neg(A \circ B)$ or solely of $B, \neg(A \circ B)$. Now we can put $v(A)=0, v(B)=0$ and $v(A \circ B)=1$, but it shows that the scheme is falsifiable, contrary to the assumption. The reasoning is analogous if 12 or 13 fails.

From $(\rightarrow)$ and $(\leftarrow): f_{\circ}(0,0)=0$ iff $\mathcal{L}_{000}$ contains correct schemes.

The reasoning is analogous for the remaining entries.

Having the 8 lists of schemes for the 8 variants of Algorithm 1, we could perform some tasks in an automatic manner. Thus we have found all schemes such that: scheme $\left(L_{1}, L_{2}\right)$ is present on at least one of the lists and $\left(L_{2}, L_{1}\right)$ is present on at least one of the lists, where these may be two different lists. The result contains all invertible rules we aimed at, and it was written in: results $[0,1,2,3,4,5,6,7]$.txt. There are 104 schemes on the list, but one should remember that for each scheme, its "inverse" is also present on the list, thus there are 52 different invertible rule schemes.

Next, we have found all schemes such that: (i) scheme $\left(L_{1}, L_{2}\right)$ is on at least one of the lists no.: $i, j, k, l$, and scheme $\left(L_{2}, L_{1}\right)$ is on at least one of these lists, where the combination $i, j, k, l$ characterizes one of the 16 different binary Boolean functions. For example, disjunction is characterized by entries '000', '011', '101', '111'; the rules corresponding to them are contained in lists nos $0,3,5$, and 7 , so the invertible rules for $\vee$ were found among these lists. The result is written in the file named $i j k l . t x t ;$ in the case of disjunction it is 0357 .txt. Here we summarise the result in Table 3. In the first column's header we put: 2-f-scheme, by which we mean a rule scheme composed of two sequents with 2 distinguished formulas each; analogously for a 3-f-scheme, where each of the two sequents has 3 distinguished formulas.

By and large, each 2-f-scheme found automatically is equivalent to one of those already listed above in Theorem 1, modulo negation expressed by a side of a sequent; or is syntactically different from them, but semantically equivalent to one of the rules in the sense that it corresponds to the same pair of entries. The situation is slightly different in the case of 3 -f-schemes: 
Table 3. invertible rules for disjunction

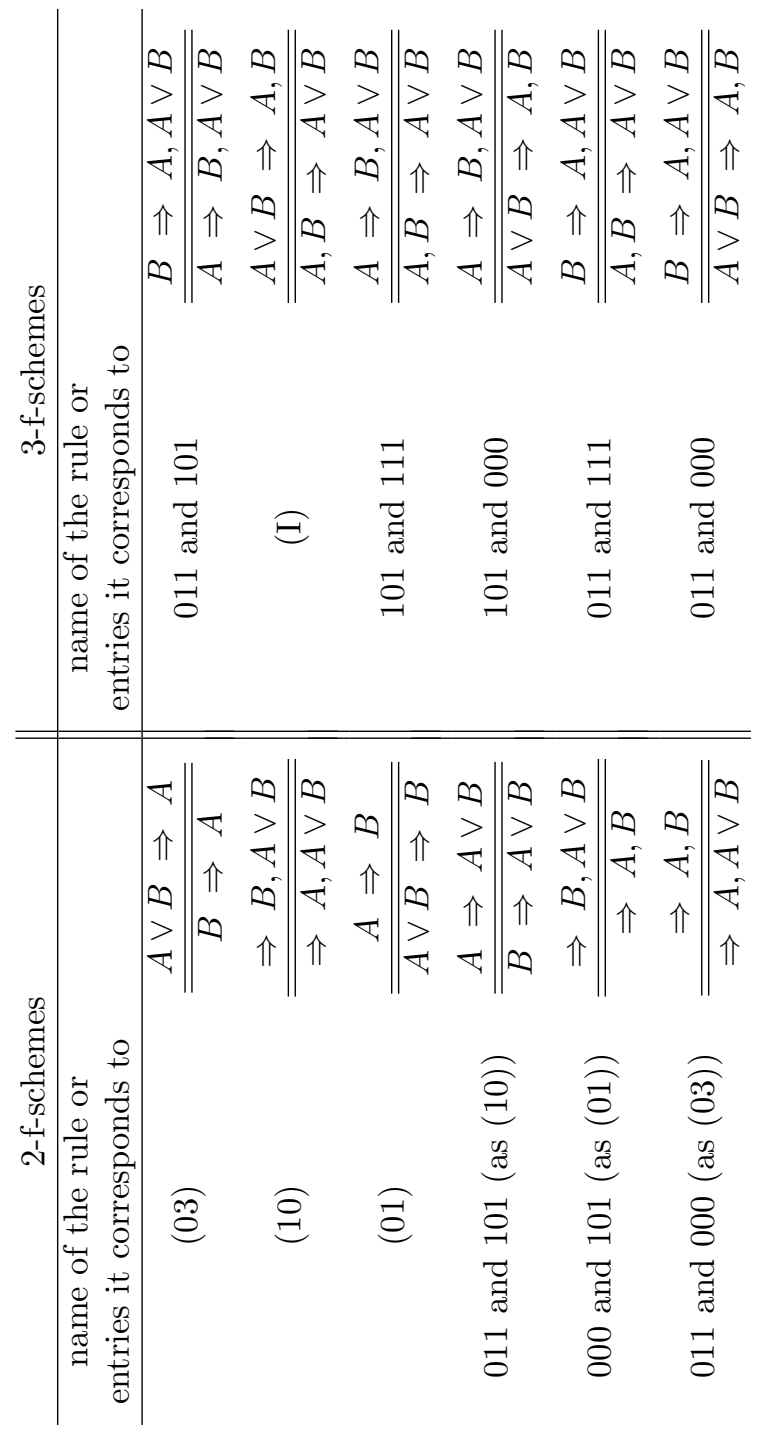


here we have found automatically more then we have previously reported in Corollary 1.

The results concerning all binary connectives are summarized in Table 4. Interestingly, the lists differ with respect to their size, where by size of a list we mean the number of schemes ${ }^{5}$ on the list, and there is a clear correspondence between a list's size and the semantic characteristics of o in terms of functional completeness, which we have introduced at the beginning of this paper.

Rules matching no connective. The next task was to find all schemes such that: (i) scheme $\left(L_{1}, L_{2}\right)$ is on at least one of the lists: 0,1 ; and (ii) so is $\left(L_{2}, L_{1}\right)$. Similarly for the lists: 2 and 3, 4 and 5, 6 and 7 . In each case, the two lists contain rules corresponding to incompatible entries, and we were curious if we can find a non-empty list as a result ... Well, yes, we can! In each case, the result is one invertible rule (see Table 5).

The leftmost rule in Table 5 is suitable when o represents a Boolean function such that $f_{\circ}(0,0)=0$ and $f_{\circ}(0,0)=1$ at the same time. Plainly, it does not make sense on the grounds of $\mathbf{C P L}$, but it reveals the potential of the automatic approach in the field of non-classical logics.

3-f-schemes. There are exactly 8 different sequents containing exactly 3 different formulas $A, B, A \circ B$ (the order of formulas in the antecedent / succedent is not essential). Each of the sequents is correct iff one of the 8 entries holds. The sequents may be combined into inference schemes in exactly $8 \cdot 8=64$ ways, among which 8 have the same premise and conclusion. Among the 56 schemes left there are 8 schemes matching no connective: those displayed in Table 5 and their inverses. Thus there are 483 -f-schemes left, hence $48 / 2=24$ different invertible rules.

On the other hand, a two-argument Boolean function is characterized by 4 entries. Each entry corresponds to exactly one sequent with 3 formulas. Hence, each such function can be assigned exactly $4 \cdot 3=123$-f-schemes. This is what we can see in Table 4: the functions differ in the numbers of the 2-f-schemes. In other words, the number of 2 -f-schemes provides a syntactic characteristics of the properties of being primary, non-primary and non-trivial, and non-primary and trivial.

Question. We have observed previously that we cannot find a match

\footnotetext{
${ }^{5}$ Let us recall that scheme is a pair $\left(L_{1}, L_{2}\right)$ and each pair goes with its inverse which forms a separate scheme. Thus in order to obtain the number of different invertible rules present on a list we need to divide the list's size by 2 .
} 
Table 4. Results for 16 Boolean functions

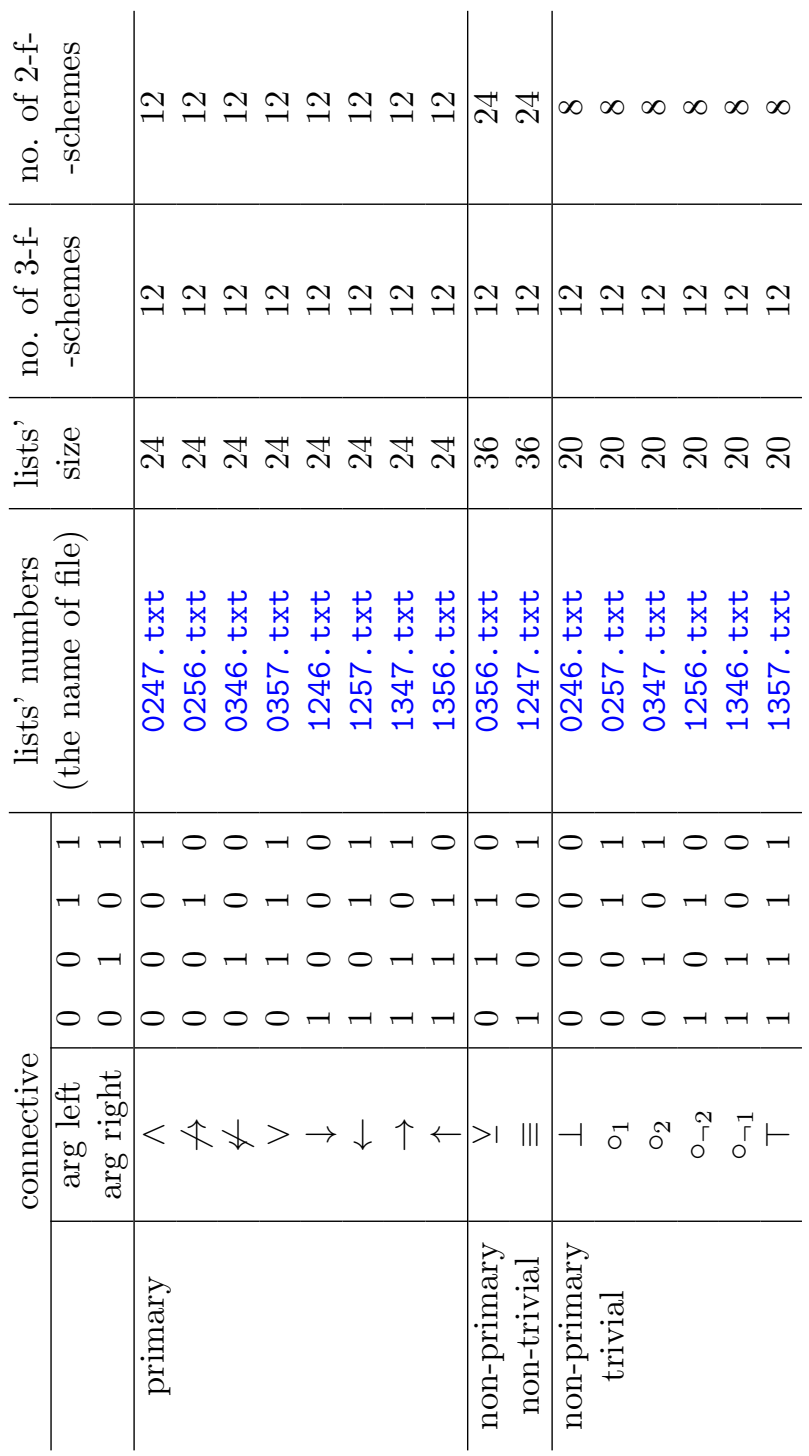




\section{Table 5 .}

$$
\begin{aligned}
& \text { lists } 0,1 \\
& \text { lists } 2,3 \\
& \text { lists } 4,5 \\
& \text { lists } 6,7 \\
& \frac{\Rightarrow A, B, A \circ B}{\overline{A \circ B \Rightarrow A}} \\
& \frac{B \Rightarrow A, A \circ B}{\overline{B, A \circ B \Rightarrow A}} \\
& \frac{A \Rightarrow B, A \circ B}{\overline{\overline{A, A \circ B \Rightarrow B}}} \\
& \frac{A, B \Rightarrow A \circ B}{\overline{A, B, A \circ B \Rightarrow}}
\end{aligned}
$$

of 2-f-schemes that would characterize a primary connective (see page 53). Now we are in a position to ask why it is the case, and to provide a sketch of the answer. When one analyses correctness of a rule top-down the reasoning may go as follows: suppose the premise is true and the conclusion is false; both the premise and conclusion contain two formulas. If the rule is correct top-down, then assigning value 0 to the two elements of the conclusion, value 1 to one element of the premise, and calculating the value of $A \circ B$ leads to a contradiction. The other element of the premise must be identical to one of the conclusion's, hence its value is settled to 0 - otherwise there would be no contradiction.

To sum up: from the six basic elements: $A, B, A \circ B, \neg A, \neg B, \neg(A \circ B)$ that occur in the scheme, there is exactly one common to the premise and the conclusion (one can also check this by inspection on invertible 2-f-schemes!). When the top-down-reasoning is conducted, the values of the other two formulas are settled by the assumption that the premise is true and the conclusion false. But the same rule is also correct bottom-up, with the same kind of reasoning settling the correctness. When the reasoning is conducted bottom-up, then the formula common to the premise and the conclusion has the same value as in the previous reasoning, and the other two are assigned values opposed to those assigned in the previous reasoning, as now the premise is assumed false, and the conclusion true. Hence it follows that:

Corollary 2. Let $\mathcal{S}=\left(L_{1}, L_{2}\right)$ be a 2 -f-scheme. If $\mathcal{S}$ corresponds to an entry $\mathscr{E}_{1}$ (top-down), and $\left(L_{2}, L_{1}\right)$ corresponds to an entry $\mathscr{E}_{2}$ (bottom-up), then the two entries differ from each other in exactly two positions.

The graph below illustrates how the entries are linked by the invertible rules. Needless to say, $i j k$ is for $f_{\circ}(i, j)=k$. As we can see, the entries create two disjoint graphs, both are full. In each case, there are exactly two "steps" in Gray code between any two nodes in the graph. 


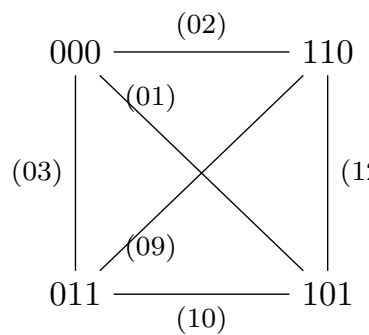

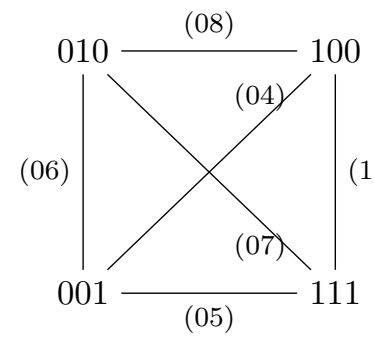

$(11)$

As we have already observed, characterizing disjunction by 2 -f-schemes requires linking entries $000,011,101,111$. We can see from the graph, however, that it is not possible. The situation is similar with the other primary connectives: being primary consists in having three 1 s and one 0 , or three $0 \mathrm{~s}$ and one 1 (in the truth table of the connective). We can see from the graphs above that it leaves us always with three edges from one of the two graphs, and that one entry is not captured by the edges. We can also observe that the 3 edges correspond to 12 2-f-schemes (see Table 4), thus each edge is represented by 2 pairs of 2 -f-schemes.

The non-primary connectives have either four $1 \mathrm{~s}$, or four $0 \mathrm{~s}$, or two $1 \mathrm{~s}$ and two 0s. Now the number of the 2 -f-schemes depends on the way the entries are linked by the edges. For example, for $\equiv$ we have:

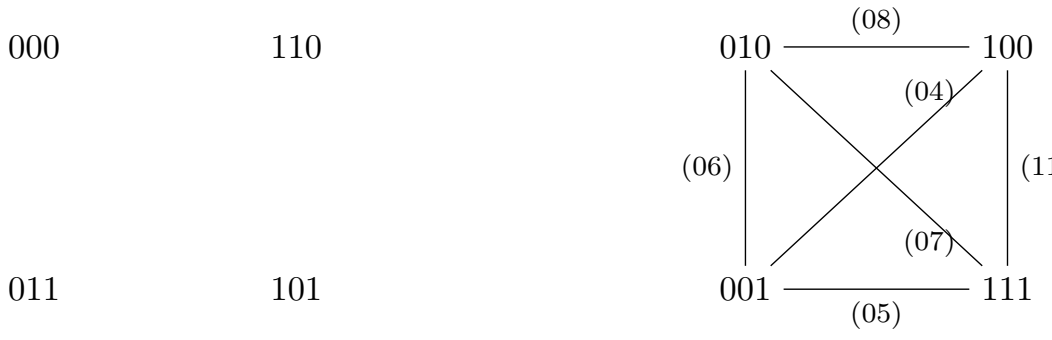

And for $\circ_{1}$ we have: 
000

011
110

101
010 100

To sum up, the non-primary and non-trivial connectives are those characterized by 6 edges in the graph and the non-primary trivial connectives are those characterized by 2 edges. Since each edge may be represented by four 2 -f-schemes (a pair of invertible 2 -f-scheme rules), this gives us $6^{*} 4=24$ and $2 * 4=82$-f-schemes in Table 4 .

\section{Soundness and completeness of the calculi}

In this section we define calculi for various fragments of CPL and demonstrate that they are sound and complete.

Let $\mathscr{C}_{N}$ (where $N$ is for $N$ on-primary) stand for a set of rules containing all the instances of $(\mathrm{Ax}),(\Rightarrow \neg),(\neg \Rightarrow)$ and (cut), and all the instances of at least one of the following:

- rules $R_{\circ}^{(02)}, R_{\circ}^{(08)}$ for $\circ_{\perp} ; \quad$ - rules $R_{\circ}^{(05)}, R_{\circ}^{(10)}$ for $\circ_{\top}$;

- rules $R_{\circ}^{(01)}, R_{\circ}^{(07)}$ for $\circ_{1}$; - rules $R_{\circ}^{(04)}, R_{\circ}^{(09)}$ for $\circ_{\neg 1}$;

- rules $R_{\circ}^{(03)}, R_{\circ}^{(11)}$ for $\circ_{2}$; $\quad$ - $R_{\circ}^{(06)}, R_{\circ}^{(12)}$ for $\circ_{\neg 2}$;

- rules $R_{\circ}^{(01)}, R_{\circ}^{(09)} /$ or $R_{\circ}^{(02)}, R_{\circ}^{(10)} /$ or $R_{\circ}^{(03)}, R_{\circ}^{(12)}$ for $\underline{\vee}$;

- rules $R_{\circ}^{(04)}, R_{\circ}^{(07)} /$ or $R_{\circ}^{(05)}, R_{\circ}^{(08)} /$ or $R_{\circ}^{(06)}, R_{\circ}^{(11)}$ for $\equiv$;

where:

$$
\begin{aligned}
& R_{\circ}^{(01)} \frac{A, \Gamma \Rightarrow \Delta, B}{A \circ B, \Gamma \Rightarrow \Delta, B} \quad R_{\circ}^{(02)} \frac{B, \Gamma \Rightarrow \Delta, \neg(A \circ B)}{A \circ B, \Gamma \Rightarrow \Delta, A} \\
& R_{\circ}^{(03)} \frac{B, \Gamma \Rightarrow \Delta, A}{A \circ B, \Gamma \Rightarrow \Delta, A} \quad R_{\circ}^{(04)} \frac{A, \Gamma \Rightarrow \Delta, B}{\neg B, \Gamma \Rightarrow \Delta, A \circ B} \\
& R_{\circ}^{(05)} \frac{B, \Gamma \Rightarrow \Delta, A \circ B}{\neg A, \Gamma \Rightarrow \Delta, A \circ B} \quad R_{\circ}^{(06)} \frac{A \circ B, \Gamma \Rightarrow \Delta, A}{\neg A, \Gamma \Rightarrow \Delta, B} \\
& R_{\circ}^{(07)} \frac{B, \Gamma \Rightarrow \Delta, A \circ B}{B, \Gamma \Rightarrow \Delta, A} \quad R_{\circ}^{(08)} \frac{A \circ B, \Gamma \Rightarrow \Delta, B}{=A \circ B, \Gamma \Rightarrow \Delta, A}
\end{aligned}
$$




$$
\begin{aligned}
& R_{\circ}^{(09)} \frac{B, \Gamma \Rightarrow \Delta, \neg(A \circ B)}{B, \Gamma \Rightarrow \Delta, A} \quad R_{\circ}^{(10)} \frac{\neg B, \Gamma \Rightarrow \Delta, A \circ B}{\neg A, \Gamma \Rightarrow \Delta, A \circ B} \\
& R_{\circ}^{(11)} \frac{A, \Gamma \Rightarrow \Delta, A \circ B}{A, \Gamma \Rightarrow \Delta, B} \quad R_{\circ}^{(12)} \frac{A, \Gamma \Rightarrow \Delta, \neg(A \circ B)}{A, \Gamma \Rightarrow \Delta, B}
\end{aligned}
$$

For primary connectives we set what follows. Let $\mathscr{C}_{P}^{A}(P$ stands for primary and $A$ for axiom) be a set of rules containing all the instances of $(\mathrm{Ax}),(\Rightarrow \neg),(\neg \Rightarrow)$, (cut) and all the instances of at least one of the following:

- $A_{\circ}^{(\mathrm{I})}$ and $R_{\circ}^{08}$ for $\wedge$; $\quad$ - $A_{\circ}^{(\mathrm{I})}$ and $R_{\circ}^{10}$ for $\vee$;

- $A_{\circ}^{(\mathrm{II})}$ and $R_{\circ}^{08}$ for $\downarrow ; \quad$ - $A_{\circ}^{(\mathrm{II})}$ and $R_{\circ}^{10}$ for $\uparrow$;

- $A_{\circ}^{(\mathrm{III})}$ and $R_{\circ}^{02}$ for $\nrightarrow$; $\quad$ - $A_{\circ}^{(\mathrm{III})}$ and $R_{\circ}^{05}$ for $\leftarrow$;

- $A_{\circ}^{(\mathrm{IV})}$ and $R_{\circ}^{02}$ for $\psi ; \quad \bullet A_{\circ}^{(\mathrm{IV})}$ and $R_{\circ}^{05}$ for $\rightarrow$.

Further, let $\mathscr{C}_{P}^{R}(R$ stands for rules $)$ be constructed analogously to $\mathscr{C}_{P}^{A}$, but with $R_{\circ}^{(\mathrm{I})}$ instead of $A_{\circ}^{(\mathrm{I})}, R_{\circ}^{(\mathrm{II})}$ instead of $A_{\circ}^{(\mathrm{II})}$, and so on. On the margin, let us observe that dual connectives (e.g., $\wedge$ and $\vee$ ) are characterised by the same axiom/rule among I-IV.

Let $\mathscr{C} \in\left\{\mathscr{C}_{N}, \mathscr{C}_{P}^{A}, \mathscr{C}_{P}^{R}\right\}$. The following concepts are needed (definitions after $[15$, p. 30]):

Definition 3. A derivation in $\mathscr{C}$ is either an instance of $(\mathrm{Ax})$ or an application of a rule of $\mathscr{C}$ to derivations concluding its premise(s). The height of a derivation is the greatest number of successive applications of rules in it, where an instance of $(\mathrm{Ax})$ has height $0 .{ }^{6}$ The height of an application of a rule $(r)$ in a derivation is the height of the subderivation leading to the conclusion of $(r)$.

We follow a standard definition of a proof in sequent calculi. For example, sequent $\Rightarrow p \equiv p$ is provable in a calculus $\mathscr{C}_{N}$ containing rules $R_{\equiv}^{(04)}$ and $R_{\equiv}^{(07)}$, and here is a proof (the arrows indicate in which direction the rule was used):

\footnotetext{
${ }^{6}$ It is almost an exact quotation of the definition in [15]. By "successive applications" the authors mean applications of rules on the same branch. An account from [28]: a derivation is a labelled tree, with labels regulated by the rules, and the height of a finite derivation is the maximum length of its branches, where the length of a branch is the number of nodes of the branch minus 1 .
} 


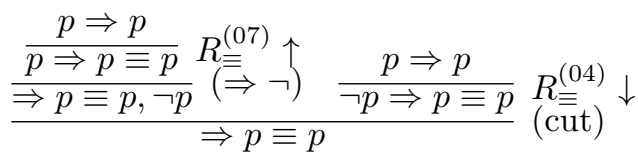

Let us now prove the following structural results:

Proposition 1. The rule of the cut is not eliminable in $\mathscr{C}_{N}$ for $\equiv$ containing rules $R_{\circ}^{(04)}, R_{\circ}^{(07)}$.

Proof: The above proof of sequent: $\Rightarrow(p \equiv p)$ delivers the argument: among the rules of $\mathscr{C}_{N}$, the sequent can fit only the conclusion of (cut).

After we prove completeness of the calculi, the above argument can be generalised as follows. Suppose that $F$ is a valid formula of $\mathscr{L}_{\neg}^{\circ}$. Then the sequent $\Rightarrow F$ is provable in a respective $\mathscr{C}$, but the sequent fits only the conclusion of (cut). Similarly, if $F$ is an inconsistent formula, then sequent $F \Rightarrow$ is provable, but only with the use of (cut). The presented argument does not work for calculi pertaining to the fragments of CPL expressed in $\left\langle\mathscr{P},\left\{\circ_{1}, \circ_{2}, \circ_{\neg 2}, \circ_{\neg 1}\right\}, \neg,(),\right\rangle$, for these fragments have neither valid nor inconsistent formulas.

THEOREM 3 (admissibility of weakening). Let $F$ be a formula of $\mathscr{L}_{\neg}^{\circ}$. If $\Gamma \Rightarrow \Delta$ is provable in $\mathscr{C}$, then $F, \Gamma \Rightarrow \Delta$ and $\Gamma \Rightarrow \Delta, F$ are provable in $\mathscr{C}$. Proof: The proof follows standard constructions, like in [15, Section 2.3. Proof Methods for Admissibility]: we show by induction that the "weakenings" of a provable sequent may be "pushed upwards" from the root to the leafs.

Thus suppose that $\Gamma \Rightarrow \Delta$ is provable in $\mathscr{C}$, and let $\mathbf{p}$ stand for a proof of the sequent. The reasoning is by induction with respect to the height of $\mathbf{p}$.

Base step. If the height is 0 , then $\Gamma \Rightarrow \Delta$ is an axiom; then the same pertains to $F, \Gamma \Rightarrow \Delta$ and $\Gamma \Rightarrow \Delta, F$.

Induction step. Height of $\mathbf{p}$ is $n \geq 1$. Now we consider the rule which concludes $\Gamma \Rightarrow \Delta$. Suppose the rule is $R_{\circ}^{(01)}$. Then $\mathbf{p}$ ends with:

$$
\frac{\frac{\vdots}{A, \Gamma^{*} \Rightarrow \Delta^{*}, B}}{A \circ B, \Gamma^{*} \Rightarrow \Delta^{*}, B} R_{\circ}^{(01)}
$$

where $\Gamma=A \circ B, \Gamma^{*}$ and $\Delta=\Delta^{*}, B$. Then a derivation concluding $A, \Gamma^{*} \Rightarrow \Delta^{*}, B$ is of height $n-1$, and by induction hypothesis, sequents 
$F, A, \Gamma^{*} \Rightarrow \Delta^{*}, B$ as well as $A, \Gamma^{*} \Rightarrow \Delta^{*}, B, F$ are provable in $\mathscr{C}$. But then an application of $R_{\circ}^{(01)}$ leads to a proof of sequent $F, \Gamma \Rightarrow \Delta$, or, respectively, sequent $\Gamma \Rightarrow \Delta, F$. The reasoning is analogous in the case of the other one-premise rules.

Thus suppose that $\Gamma \Rightarrow \Delta$ results by (cut):

$$
\frac{\frac{\vdots}{\Gamma \Rightarrow \Delta, A} \frac{\vdots}{A, \Gamma \Rightarrow \Delta}}{\Gamma \Rightarrow \Delta} \text { (cut) }
$$

The premises of (cut) both have proofs of length $n-1$, thus, by induction hypothesis, sequents $F, \Gamma \Rightarrow \Delta, A$ and $\Gamma \Rightarrow \Delta, A, F$, and also $F, A, \Gamma \Rightarrow \Delta$ and $A, \Gamma \Rightarrow \Delta, F$ are provable in $\mathscr{C}$. Then:

$$
\frac{\frac{\vdots}{F, \Gamma \Rightarrow \Delta, A} \quad \frac{\vdots}{A, F, \Gamma \Rightarrow \Delta}}{F, \Gamma \Rightarrow \Delta} \text { (cut) }
$$

shows how to prove $F, \Gamma \Rightarrow \Delta$, and similarly for $\Gamma \Rightarrow \Delta, F$.

We state the following without a proof.

Proposition 2. If a sequent, $\varphi$, of language $\mathscr{L}_{\neg \Rightarrow}^{\circ}$ is of the form (Ax), then $=\varphi$.

TheOrem 4 (Soundness). For each sequent $\Gamma \Rightarrow \Delta$ of language $\mathscr{L}_{\neg \Rightarrow}^{\circ}$, if $\vdash_{\mathscr{C}} \Gamma \Rightarrow \Delta$, then $=\Gamma \Rightarrow \Delta$.

Proof: By induction on the height of a proof. Use Proposition 2, Theorem 1 , and Theorem 2 (for the case of $\mathscr{C}_{P}^{A}$ ) or Corollary 1 (for the case of $\mathscr{C}_{P}^{R}$ ). One can also use Lemma 1 for the automatically generated rules.

Corollary 3 (Soundness for formulas). For each $A \in \mathscr{F}_{\neg}^{\circ}$, it holds that if $\Rightarrow A$ is provable in $\mathscr{C}$, then $=A$.

Proof: Immediately follows from Theorem 4.

Completeness. We prove completeness theorem by Kalmár's method. We start with some auxiliary definitions and lemmas.

Definition 4. Let $A \in \mathscr{F}_{\neg}^{\circ}$ and $v$ be an arbitrary valuation. Then $A^{v}$ is $A$ 's $v$-image iff

$$
A^{v}=\left\{\begin{array}{ccc}
A & \text { iff } & v(A)=1 \\
\neg A & \text { iff } & v(A)=0 .
\end{array}\right.
$$


Lemma 2. Let $x, y, z \in\{1,0\}, A, B \in \mathscr{F}_{\neg}^{\circ}, v_{x}, v_{y}$, and $v_{z}$ be valuations such that $v_{x}(A)=x, v_{y}(B)=y$, and $v_{z}(A \circ B)=z$. Then if $f_{\circ}(x, y)=z$, then $A^{v_{x}}, B^{v_{y}} \Rightarrow(A \circ B)^{v_{z}}$ is provable in the respective $\mathscr{C}$ for $\circ$.

Proof: Consider the case $f_{\circ}(0,0)=0$. We need to show that $\neg A, \neg B \Rightarrow$ $\neg(A \circ B)$ is provable in the respective $\mathscr{C}$ for $\circ \in\left\{\circ_{\perp}, \wedge, \not \rightarrow, \circ_{1}, \forall, \circ_{2}, \underline{\vee}, \vee\right\}$. If $\circ=\circ_{1}$, then we have $R_{\circ}^{(01)}$ in the calculus, if $\circ \in\left\{\circ_{\perp}, \not \rightarrow, \nvdash\right\}$, then we have $R_{\circ}^{(02)}$, if $\circ=\circ_{2}$, then there is $R_{\circ}^{(03)}$, and if $\circ=\underline{\vee}$, then one of the three rules is present. In these cases we follow the appropriate schemes of derivation:

$$
\begin{aligned}
& \begin{array}{cl}
\frac{A \Rightarrow B, A}{\neg A, A \Rightarrow B}(\neg \Rightarrow) & \frac{B \Rightarrow \neg(A \circ B), B}{\neg B, B \Rightarrow \neg(A \circ B)}(\neg \Rightarrow) \\
\frac{\neg A, A \circ B \Rightarrow B}{\neg R_{\circ}^{(01)} \downarrow} & \frac{\neg B, A \circ B \Rightarrow A}{\neg(02)} \downarrow \\
\frac{\neg A, \neg B, A \circ B \Rightarrow}{\neg A, \neg B \Rightarrow \neg(A \circ B)}(\Rightarrow \neg) & \frac{\neg A, \neg B, A \circ B \Rightarrow}{\neg A, \neg B \Rightarrow \neg(A \circ B)}(\Rightarrow \neg)
\end{array} \\
& \begin{array}{c}
\frac{B \Rightarrow A, B}{\neg B, B \Rightarrow A}(\neg \Rightarrow) \\
\frac{\neg B, A \circ B \Rightarrow A}{\neg A, \neg B, A \circ B \Rightarrow}(\neg \Rightarrow) \\
\frac{\neg A, \neg B \Rightarrow \neg(A \circ B)}{\neg A, \neg}(\Rightarrow)
\end{array}
\end{aligned}
$$

In the case of $\circ=\{\wedge, \vee\}$, we cannot apply any of the above rules. If $\mathscr{C}_{P}=\mathscr{C}_{P}^{A}$, then we have the axiom $A_{\circ \downarrow}^{(\mathrm{I})}$, and the following shows that $\vdash_{\mathscr{C}_{P}^{A}} \neg A, \neg B \Rightarrow \neg(A \circ B)$ :

$$
\frac{\frac{A \circ B \Rightarrow A, B}{\neg A, \neg B, A \circ B \Rightarrow}(\neg \Rightarrow) \times 2}{\neg A, \neg B \Rightarrow \neg(A \circ B)}(\Rightarrow \neg)
$$

If $\mathscr{C}_{P}=\mathscr{C}_{P}^{R}$, then we have the rule $R_{\circ}^{(\mathrm{I})}$ :

$$
\begin{aligned}
& \frac{A, B \Rightarrow A, A \circ B}{A \circ B \Rightarrow A, A, B} R_{\circ}^{(\mathrm{I})} \downarrow \\
& \frac{\frac{\neg A, \neg B, A \circ B \Rightarrow A}{\neg}(\neg \Rightarrow) \times 2 \quad(\Rightarrow \neg) \quad \frac{A, \neg B \Rightarrow A, \neg(A \circ B)}{A, \neg A, \neg B \Rightarrow \neg(A \circ B)}}{(\neg \Rightarrow)} \text { (cut) }
\end{aligned}
$$

The proof is analogous in the remaining cases. To save space, we publish the remaining part of the proof in the file proof-Lemma2.pdf on https://github.com/mjukiewicz/Correspondence-Analysis. 
Lemma 3. Let $A \in \mathscr{F}_{\neg}^{\circ}$. Let $v$ be an arbitrary valuation, $\mathscr{P}(A)$ be the set of all $A$ 's propositional variables, and $q_{1}, \ldots, q_{k}$ be propositional variables such that $\mathscr{P}(A) \subseteq\left\{q_{1}, \ldots, q_{k}\right\}$. Then:

$$
q_{1}^{v}, \ldots, q_{k}^{v} \Rightarrow A^{v} \text { is provable in } \mathscr{C} \text {. }
$$

Proof: We use structural induction on $A$.

Basis. $A$ is a propositional variable. Clearly, $A \in\left\{q_{1}, \ldots, q_{k}\right\}$. Then $A^{v} \in\left\{q_{1}^{v}, \ldots, q_{k}^{v}\right\}$. Thus, $\vdash_{\mathscr{C}} q_{1}^{v}, \ldots, q_{k}^{v} \Rightarrow A^{v}$, since it is an axiom.

Induction step 1. $A=\neg B$. By the inductive hypothesis, it holds that $\vdash_{\mathscr{C}} q_{1}^{v}, \ldots, q_{k}^{v} \Rightarrow B^{v}$. Clearly, (a) either $B^{v}=B$ or $B^{v}=\neg B$.

Suppose $B^{v}=B$. Then (b) $\vdash_{\mathscr{C}} q_{1}^{v}, \ldots, q_{k}^{v} \Rightarrow B$. Since $B^{v}=B$, so $v(B)=1$. Hence, $v(\neg B)=0$. Therefore, $\left(\right.$ c) $(\neg B)^{v}=\neg \neg B$. Now we show that (d) $\vdash_{\mathscr{C}} B \Rightarrow \neg \neg B$.

$$
\begin{gathered}
\frac{B \Rightarrow B}{\neg B, B \Rightarrow} \\
\frac{B \Rightarrow \neg \neg B}{(\neg \Rightarrow)}(\Rightarrow \neg)
\end{gathered}
$$

By Theorem 3, if (b), then also $\vdash_{\mathscr{C}} q_{1}^{v}, \ldots, q_{k}^{v} \Rightarrow \neg \neg B, B$; and if (d), then also $\vdash_{\mathscr{C}} B, q_{1}^{v}, \ldots, q_{k}^{v} \Rightarrow \neg \neg B$. It follows that there exists a proof concluding the following:

$$
\frac{\vdots}{q_{1}^{v}, \ldots, q_{k}^{v} \Rightarrow \neg \neg B, B \quad B, q_{1}^{v}, \ldots, q_{k}^{v} \Rightarrow \neg \neg B} \text { (cut) }
$$

which shows provability of $q_{1}^{v}, \ldots, q_{k}^{v} \Rightarrow \neg \neg B$ in $\mathscr{C}$. From the latter and (c) we obtain (e) $\vdash_{\mathscr{C}} q_{1}^{v}, \ldots, q_{k}^{v} \Rightarrow(\neg B)^{v}$. Recall that $A=\neg B$. Then, by (e), $\vdash_{\mathscr{C}} q_{1}^{v}, \ldots, q_{k}^{v} \Rightarrow A^{v}$. Thus, (f) if $B^{v}=B$, then $\vdash_{\mathscr{C}} q_{1}^{v}, \ldots, q_{k}^{v} \Rightarrow A^{v}$.

Suppose $B^{v}=\neg B$. Then $\vdash_{\mathscr{C}} q_{1}^{v}, \ldots, q_{k}^{v} \Rightarrow \neg B$ and $(\neg B)^{v}=\neg B .^{7}$ Clearly $\vdash_{\mathscr{C}} q_{1}^{v}, \ldots, q_{k}^{v} \Rightarrow(\neg B)^{v}$. Hence, $\vdash_{\mathscr{C}} q_{1}^{v}, \ldots, q_{k}^{v} \Rightarrow A^{v}$. Thus, (g) if $B^{v}=\neg B$, then $\vdash_{\mathscr{C}} q_{1}^{v}, \ldots, q_{k}^{v} \Rightarrow A^{v}$.

By (a), (f), and (g), $\vdash \mathscr{C} q_{1}^{v}, \ldots, q_{k}^{v} \vdash A^{v}$.

Induction step 2. $A=B \circ C$. By the inductive hypothesis, it holds that $\vdash_{\mathscr{C}} q_{1}^{v}, \ldots, q_{k}^{v} \Rightarrow B^{v}$ and $\vdash_{\mathscr{C}} q_{1}^{v}, \ldots, q_{k}^{v} \Rightarrow C^{v}$. By Theorem 3, also $\vdash_{\mathscr{C}} C^{v}, q_{1}^{v}, \ldots, q_{k}^{v} \Rightarrow(B \circ C)^{v}, B^{v}$ and $\vdash_{\mathscr{C}} q_{1}^{v}, \ldots, q_{k}^{v} \Rightarrow(B \circ C)^{v}, C^{v}$. Let (h) $f_{\circ}(x, y)=z$. Thus, (i) if $v(B)=x$ and $v(C)=y$, then $v(B \circ C)=z$. Let $(\mathrm{j}) v_{x}, v_{y}$, and $v_{z}$ be valuations such that $v(B)=v_{x}(B), v(C)=v_{y}(C)$, and $v(B \circ C)=v_{z}(B \circ C)$. Then $(\mathrm{k}) B^{v}=B^{v_{x}}, C^{v}=C^{v_{y}}$, and $(B \circ C)^{v}=$ $(B \circ C)^{v_{z}}$.

${ }^{7}$ Observe that if $v(B)=0$, then $(\neg B)^{v}=\neg B$, but $\neg(B)^{v}=\neg(\neg B)=\neg \neg B$. 
Suppose (l) $v(B)=x$ and $v(C)=y$. Then, by (i), it holds that $(\mathrm{m}) v(B \circ C)=z$. By $(\mathrm{h}),(\mathrm{j}),(\mathrm{l}),(\mathrm{m})$, and Lemma 2, we obtain $\vdash_{\mathscr{C}}$ $B^{v_{x}}, C^{v_{y}} \Rightarrow(B \circ C)^{v_{z}}$. Then, by $(\mathrm{k}), \vdash_{\mathscr{C}} B^{v}, C^{v} \Rightarrow(B \circ C)^{v}$. Again, by Theorem 3 , also $\vdash_{\mathscr{C}} B^{v}, C^{v}, \Gamma \Rightarrow(B \circ C)^{v}$, where $\Gamma$ is for $q_{1}^{v}, \ldots, q_{k}^{v}$. Using the inductive hypothesis and two applications of (cut), we show that $\vdash_{\mathscr{C}} q_{1}^{v}, \ldots, q_{k}^{v} \Rightarrow(B \circ C)^{v}$ :

$$
\begin{aligned}
& \begin{array}{c}
\vdots \\
\Gamma \Rightarrow(B \circ C)^{v}, C^{v}
\end{array} \frac{C^{v}, \Gamma \Rightarrow(B \circ C)^{v}, B^{v} \quad B^{v}, C^{v}, \Gamma \Rightarrow(B \circ C)^{v}}{C^{v}, \Gamma \Rightarrow(B \circ C)^{v}} \\
& \Gamma \Rightarrow(B \circ C)^{v}
\end{aligned}
$$

Since $A=B \circ C$, so $\vdash_{\mathscr{C}} q_{1}^{v}, \ldots, q_{k}^{v} \Rightarrow A^{v}$.

Lemma 4. Let $A \in \mathscr{F} \circ$ and $q_{1}, q_{2}, \ldots, q_{k} \in \mathscr{P}$. Then it holds that if $\vdash_{\mathscr{C}} q_{1}^{v}, q_{2}^{v}, \ldots, q_{k}^{v} \Rightarrow A$, for each valuation $v$, then $\vdash_{\mathscr{C}} q_{2}^{v}, \ldots, q_{k}^{v} \Rightarrow A$, for each valuation $v$.

Proof: Suppose (a) $\vdash_{\mathscr{C}} q_{1}^{v}, q_{2}^{v}, \ldots, q_{k}^{v} \Rightarrow A$, for each valuation $v$. Let $w$ be an arbitrary valuation. Let $u$ be a valuation such that

(i) $w(p)=u(p)$, for each $p \in \mathscr{P}$ such that $p \neq q_{1}$;

(ii) $u\left(q_{1}\right)=\left\{\begin{array}{lll}1 & \text { iff } & w\left(q_{1}\right)=0 \\ 0 & \text { iff } & w\left(q_{1}\right)=1\end{array}\right.$

By (i), it holds that (b) $\left\langle q_{2}^{w}, \ldots, q_{k}^{w}\right\rangle=\left\langle q_{2}^{u}, \ldots, q_{k}^{u}\right\rangle$. By (a), it holds that (c) $\vdash_{\mathscr{C}} q_{1}^{w}, q_{2}^{w}, \ldots, q_{k}^{w} \Rightarrow A$ and (d) $\vdash_{\mathscr{C}} q_{1}^{u}, q_{2}^{u}, \ldots, q_{k}^{u} \Rightarrow A$. By (b) and (d), it holds that (e) $\vdash_{\mathscr{C}} q_{1}^{u}, q_{2}^{w}, \ldots, q_{k}^{w} \Rightarrow A$. (c) and (e) together entail: $\vdash_{\mathscr{C}} q_{1}, q_{2}^{w}, \ldots, q_{k}^{w} \Rightarrow A$ and $\vdash_{\mathscr{C}} \neg q_{1}, q_{2}^{w}, \ldots, q_{k}^{w} \Rightarrow A$.

We show that if $\vdash_{\mathscr{C}} q_{1}, \Gamma \Rightarrow A$ and $\vdash_{\mathscr{C}} \neg q_{1}, \Gamma \Rightarrow A$, then $\vdash_{\mathscr{C}} \Gamma \Rightarrow A$.

$$
\begin{array}{cc}
\vdots & \\
\frac{q_{1}, \Gamma \Rightarrow A}{\Gamma \Rightarrow A, \neg q_{1}}(\Rightarrow \neg) & \vdots \\
& \Gamma \Rightarrow q_{1}, \Gamma \Rightarrow A
\end{array} \text { (cut) }
$$

Thus, if (c) and (e), then $\vdash_{\mathscr{C}} q_{2}^{w}, \ldots, q_{k}^{w} \Rightarrow A$. Since $w$ is an arbitrary valuation, so $\vdash_{\mathscr{C}} q_{2}^{v}, \ldots, q_{k}^{v} \Rightarrow A$, for each valuation $v$.

Lemma 5. For each $A \in \mathscr{F}_{\neg}^{\circ}$ and $q_{1}, \ldots, q_{k} \in \mathscr{P}$ such that $q_{i} \neq q_{j}$, for each $i, j \in\{1, \ldots, k\}$ such that $i \neq j$, it holds that: if $\vdash_{\mathscr{C}} q_{1}^{v}, \ldots, q_{k}^{v} \Rightarrow A$, for each valuation $v$, then $\Rightarrow A$ is provable in $\mathscr{C}$.

Proof: By a straightforward induction on $k$. 
Basis. We need to show that if $\vdash_{\mathscr{C}} q^{v} \Rightarrow A$, for each valuation $v$ and $q \in \mathscr{P}$, then $\Rightarrow A$ is provable in $\mathscr{C}$.

Suppose $\vdash_{\mathscr{C}} q^{v} \Rightarrow A$, for each valuation $v$ and $q \in \mathscr{P}$. By Lemma 4, $\vdash_{\mathscr{C}} \Rightarrow A$, for each valuation $v$ and $q \in \mathscr{P}$, i.e. $\Rightarrow A$ is provable in $\mathscr{C}$.

Induction step. By the inductive hypothesis, for each valuation $v$ and $q_{1}, \ldots, q_{m} \in \mathscr{P}$ such that $q_{i} \neq q_{j}$, for each $i, j \in\{1, \ldots, m\}$ such that $i \neq j$, it holds that if $\vdash_{\mathscr{C}} q_{1}^{v}, \ldots, q_{m}^{v} \Rightarrow A$, then $\Rightarrow A$ is provable in $\mathscr{C}$.

Let (a) $r_{1}, \ldots, r_{m}, r_{m+1} \in \mathscr{P}$ such that $r_{i} \neq r_{j}$, for each $i, j \in\{1, \ldots$, $m, m+1\}$ such that $i \neq j$, and (b) $\vdash_{\mathscr{C}} r_{m+1}^{v}, r_{1}^{v}, \ldots, r_{m}^{v} \Rightarrow A$. Then, by Lemma 4, (c) $\vdash_{\mathscr{C}} r_{1}^{v}, \ldots, r_{m}^{v} \Rightarrow A$, for each valuation $v$. By the inductive hypothesis, if (d) $\vdash_{\mathscr{C}} r_{1}^{v}, \ldots, r_{m}^{v} \Rightarrow A$, then $\Rightarrow A$ is provable in $\mathscr{C}$. By (c) and (d), it holds that (e) $\Rightarrow A$ is provable in $\mathscr{C}$. By (a) and (e), we obtain that, for each valuation $v$ and $q_{1}, \ldots, q_{m}, q_{m+1} \in \mathscr{P}$ such that $q_{i} \neq q_{j}$, for each $i, j \in\{1, \ldots, m, m+1\}$ such that $i \neq j$, it holds that if $\vdash_{\mathscr{C}} q_{m+1}^{v}, q_{1}^{v}, \ldots, q_{m}^{v} \Rightarrow A$, then $\Rightarrow A$ is provable in $\mathscr{C}$.

Theorem 5 (Completeness). For each $A \in \mathscr{F}_{\neg}^{\circ}$, it holds that if $\models A$, then $\Rightarrow A$ is provable in $\mathscr{C}$.

Proof: Suppose $\models A$. Then (a) $v(A)=1$, for each valuation $v$. Since the set $\mathscr{P}(A)$ of all $A$ 's propositional variables is finite, so there are $q_{1}, \ldots, q_{k} \in$ $\mathscr{P}$ such that $\mathscr{P}(A) \subseteq\left\{q_{1}, \ldots, q_{k}\right\}$. Then, by Lemma $3,(\mathrm{~b}) \vdash_{\mathscr{C}} q_{1}^{v}, \ldots, q_{k}^{v} \Rightarrow$ $A^{v}$. By (a) and (b), it holds that (c) $\vdash_{\mathscr{C}} q_{1}^{v}, \ldots, q_{k}^{v} \Rightarrow A$. By (c) and Lemma 5 , we obtain the conclusion that $\Rightarrow A$ is provable in $\mathscr{C}$.

By Theorem 5 and Corollary 3, we have the following Corollary 4 . Corollary 4 (Adequacy). For each $A \in \mathscr{F}_{\neg}^{\circ}, \models A$ iff $\vdash_{\mathscr{C}} \Rightarrow A$

Let $\mathscr{C}$ stand for any extension of $(\mathrm{Ax}),(\Rightarrow \neg),(\neg \Rightarrow)$ and (cut) with a collection of axioms and/or rules characterising one, or more than one, binary connective. In other words, $\mathscr{C}$ can be one of $\mathscr{C}_{N}$, one of $\mathscr{C}_{P}$, or a sum of any number of such calculi. By arguments analogous to those presented above, we can show that:

Corollary 5 (Adequacy of $\mathscr{C}$ ). For each $A \in \mathscr{F}_{\neg}^{\circ}, \models A$ iff $\vdash_{\mathscr{C}} \Rightarrow A$.

\section{Conclusions and Further Work}

This paper examines the potential of correspondence analysis in designing sequent calculi for CPL. The rules corresponding to binary connectives are always linear, so the only branching rule of the calculi is (cut). What is 
more, correspondence analysis invites automatic approach to rules' generation. This combination of sequent calculi, non-eliminable (cut), and an automatic search for rules opens the possibility of interesting results in efficient proof-search. This is one of the topics of future research.

Moreover, it seems especially appealing to use the automated account to find sequent calculi for multi-valued logics, perhaps logics for which no such calculi are known. This is another perspective for future research.

Acknowledgements. The authors' special thanks go to the audience of a research seminar at Department of Logic and Cognitive Science of Adam Mickiewicz University in Poznań.

Dorota Leszczyńska-Jasion and Marcin Jukiewicz were supported financially by Polish National Science Centre, grant no. 2017/26/E/HS1/00127.

Yaroslav Petrukhin was supported financially by Polish National Science Centre, grant no. 2017/25/B/HS1/01268.

\section{References}

[1] F. G. Asenjo, A calculus of antinomies, Notre Dame Journal of Formal Logic, vol. 7, no. 1 (1966), pp. 103-105.

[2] N. D. Belnap, A useful four-valued logic, Modern Uses of MultipleValued Logic, ed. by J.M. Dunn, G. Epstein. Boston: Reidel Publishing Company (1977), pp. 7-37.

[3] N. D. Belnap, How a computer should think, Contemporary Aspects of Philosophy, ed. by G. Rule. Stocksfield: Oriel Press (1977), pp. 30-56.

[4] S. Bonzio, J. Gil-Férez, F. Paoli, L. Peruzzi, On Paraconsistent Weak Kleene Logic: Axiomatisation and Algebraic Analysis, Studia Logica, vol. 105, no. 2 (2017), pp. 253-297.

[5] K. Došen, Logical constants as punctuation marks, Notre Dame Journal of Formal Logic, vol. 30, no. 3 (1989), pp. 362-381.

[6] J. M. Dunn, Intuitive semantics for first-degree entailment and coupled trees, Philosophical Studies, vol. 29, no. 3 (1976), pp. 149-168.

[7] M. Fitting, First-Order Logic and Automated Theorem Proving, New York: Springer-Verlag, 1990.

[8] S. Halldén, The Logic of Nonsense. Lundequista Bokhandeln, Uppsala, 1949. 
[9] A. Indrzejczak, Rule-Generation Theorem and Its Applications, Bulletin of the Section of Logic, vol. 47, no. 4 (2018), pp. 265-281.

[10] A. Karpenko, N. Tomova, Bochvar's three-valued logic and literal paralogics: Their lattice and functional equivalence, Logic and Logical Philosophy, vol. 26 , no. 2 (2017), pp. 207-235.

[11] S. C. Kleene, On a notation for ordinal numbers, The Journal of Symbolic Logic, vol. 3, no. 1 (1938), pp. 150-155.

[12] S. C. Kleene, Introduction to metamathematics, Sixth Reprint, Wolters-Noordhoff Publishing and North-Holland Publishing Company, 1971.

[13] B. Kooi, A. Tamminga, Completeness via correspondence for extensions of the logic of paradox, The Review of Symbolic Logic, vol. 5, no. 4 (2012), pp. $720-730$.

[14] E. Kubyshkina, D. V. Zaitsev, Rational agency from a truth-functional perspective, Logic and Logical Philosophy, vol. 25, no. 4 (2016), pp. 499520 .

[15] S. Negri, J. von Plato, Structural Proof Theory, Cambridge: Cambridge University Press, 2001.

[16] Y. Petrukhin, V. Shangin, Automated correspondence analysis for the binary extensions of the logic of paradox, The Review of Symbolic Logic, vol. 10, no. 4 (2017), pp. 756-781.

[17] Y. Petrukhin, V. Shangin, Automated proof searching for strong Kleene logic and its binary extensions via correspondence analysis, Logic and Logical Philosophy, vol. 28, no. 2 (2019), pp. 223-257.

[18] Y. Petrukhin, V. Shangin, Natural three-valued logics characterised by natural deduction, Logique et Analyse, vol. 244 (2018), pp. 407-427.

[19] Y. Petrukhin, V. Shangin, Completeness via correspondence for extensions of paraconsistent weak Kleene logic, The Proceedings of the 10th Smirnov Readings in Logic (2017), pp. 114-115.

[20] Y. Petrukhin, V. Shangin, Correspondence Analysis and Automated Proofsearching for First Degree Entailment, European Journal of Mathematics, online first article, DOI: 10.1007/s40879-019-00344-5.

[21] Y. I. Petrukhin, Correspondence analysis for first degree entailment, Logical Investigations, vol. 22, no. 1 (2016), pp. 108-124.

[22] Y. Petrukhin, Generalized Correspondence Analysis for Three-Valued Logics, Logica Universalis, vol. 12, no. 3-4 (2018), pp. 423-460.

[23] Y. I. Petrukhin, Correspondence analysis for logic of rational agent, Chelyabinsk Physical and Mathematical Journal, vol. 2, no. 3 (2017), pp. 329-337. 
[24] G. Priest, The logic of paradox, Journal of Philosophical Logic, vol. 8, no. 1 (1979), pp. 219-241.

[25] K. Segerberg, Arbitrary truth-value functions and natural deduction, Mathematical Logic Quarterly, vol. 29, no. 11 (1983), pp. 557-564.

[26] A. Tamminga, Correspondence analysis for strong three-valued logic, Logical Investigations, vol. 20 (2014), pp. 255-268.

[27] N. E. Tomova, A lattice of implicative extensions of regular Kleene's logics, Reports on Mathematical Logic, vol. 47 (2012), pp. 173-182.

[28] A. S. Troelstra, H. Schwichtenberg, Basic Proof Theory, second edition, Cambridge: Cambridge University Press, 2000.

[29] W. Wernick, Complete sets of logical functions, Transactions of the American Mathematical Society, vol. 51 (1942), pp. 117-132.

Department of Logic and Cognitive Science, Adam Mickiewicz University, Poznań, Poland e-mail: Dorota.Leszczynska@amu.edu.pl e-mail: Marcin.Jukiewicz@amu.edu.pl

Department of Logic, Faculty of Philosophy, Lomonosov Moscow State University, Moscow, Russia e-mail: petrukhin@philos.msu.ru e-mail: shangin@philos.msu.ru 